$$
\text { "actumen" — 2008/4/9 — 9:44 — page 363 — \#1 }
$$

\title{
Constructing the disk method formula for the volume obtained by revolving a curve around an axis with the help of CAS
}

\author{
Muharrem Aktümen and Tolga Kabaca
}

Abstract. Calculus concepts should have been taught in a carefully designed learning environment, because these concepts constitute a very important base for almost all applied sciences. The integral, one of the fundamental concepts of Calculus, has a wide application area. This paper focuses on constructing the disk method formula for the volume obtained by revolving a curve around an axis with the help of a CAS.

In this study, a semi-structured interview was carried out. In this interview, we tried to construct the disk method formula.

The levels of constructing the disk method formula in this study are:

- Introducing the concept: evaluating the volume of an Egyptian pyramid.

- Evaluating the volume of a cone obtained by revolution (using Maple worksheet).

- Designing their own ring and evaluating its price (using Maplet).

In this study, the interview has been presented as a dialog between teacher and students. When we look at feedback from students, we see that such a teaching method effects students in a positive way and causes them to gain conceptual understanding directed towards the concepts of approximation and volume.

Key words and phrases: Maple, Maplet, approximations, volume, definite integral.

ZDM Subject Classification: A05, B45, A25.

Copyright (C) 2007 by University of Debrecen 


$$
\text { "actumen" — 2008/4/9 — 9:44 — page 364 — \#2 }
$$

\section{Introduction}

Computer algebra systems were originally created for use by applied mathematicians and engineers. Since their development in the 1970's and their introduction into some tertiary teaching and learning in the 1980's, Computer Algebra Systems (CAS) have been recognized as highly valuable for doing mathematics and potentially valuable for teaching and learning mathematics [1]. Today, the most popular systems are: Maple, Mathematica, Reduce, and Derive.

Clements [2] identified a number of main roles which a CAS can fulfill.

1. A mathematical laboratory or toolkit: Mathematical and allied practitioners use CASs to help explore new ideas and new mathematical structures. The features of CAS which are important to such users are the ability to define new mathematical entities and new operations on such entities. These features are essentially those of the CAS as a mathematical programming language and an extensible system.

2. A mathematical assistant: Mathematically skilled scientists, engineers and allied practitioners often need to carry out computations using wellestablished methods but of a magnitude and/or complexity which would be too difficult, costly or time-consuming by traditional pencil and paper methods. A CAS can act as a tireless, quick and (usually) impeccably accurate mathematical assistant in such situations. Not only do CASs help with calculations which would otherwise be too tedious and time-consuming but their very existence encourages users to contemplate computations which they would otherwise have rejected as impractical.

3. A mathematical expert system: Mathematically less well qualified users can call upon CASs to carry out manipulations which they might not feel confident to complete by hand for lack of manipulative skill. In other circumstances such users might have consulted a more mathematically skilled person for guidance and validation of their mathematical activities. Thus, for such users, the CAS is acting as a mathematical advisor or expert. Of course, it is important that such users are aware of the possibilities which such use brings for the introduction of inadvertent errors and they must learn to implement routinely appropriate error checking and validation strategies. 


$$
\text { "actumen" — 2008/4/9 — 9:44 — page 365 — \#3 }
$$

\section{CAS based on Constructivist Approaches in Mathematics Education}

We are all aware of the importance of constructivist approaches in mathematics education. Imagine a mathematics course! "The texts are well structured, nicely formatted and polished, and offer little leeway both for the teacher and the learner. Definitions and theorems are well structured and easily understandable. The teacher has enough both mathematical and pedagogical knowledge to transfer his or her information to the students". Even if there is no problem for almost everyone in this environment, most of the time the historical background of mathematical concepts is forgotten.

Generally, the end users are not aware that the build-up of mathematics has always been a creative process which most of the time involves lost of experimentation. We don't realize how much hard work was done by great mathematicians in the past. Students tend to think mathematical discovery only comes by stroke of genius. Students are convinced that they will never have such luck, and this adds the general uneasiness about mathematics and its teaching.

Many educators therefore want to follow a traditional paradigm using sequence "Definition $\rightarrow$ theorem $\rightarrow$ proof $\rightarrow$ corollary $\rightarrow$ application" by an approach which is more historic using the discovery chain "Problem $\rightarrow$ experiment $\rightarrow$ conjecture and idea of proof" [4].

In a technology-based constructivist learning environment, students can find the opportunity to discover, making conjectures and constructing their own mathematical knowledge. Many researchers used a CAS like Maple, Mathematica or Derive making students to discover by visualizing the calculus concepts ([5], [9], [10], [11], [12]).

A CAS allows lots of experimenting by the students, thus helping to find reasonable conjectures $([5],[6])$. As a result of a great body of research, it can be understood that using CAS is more meaningful when it was integrated with constructivist principles in the same environment ([7], [8]).

Considering all of these points, in this paper we found that a CAS can have an effect on a discovery application in an advanced calculus course.

\section{Problem Situation}

Calculus concepts should have been taught in a carefully designed learning environment, because these concepts constitute a very important base for almost all applied sciences. 
The integral, one of the fundamental themes of Calculus, has a wide application area. In the web site of the Turkish Language Association, the definitions of the concept of the integral, "The total consists of pieces" and "a (mathematical) function whose derivative is known," are given. The first meaning includes finding the areas under curves, calculating the volume of various material things, and other application areas, and is called the definite integral. This paper focuses on calculating the volume of various material things through the integral.

The concept of volume is utilized in a wide variety of applications including the physical sciences and all of the engineering disciplines. For example, in constructing a highway one uses survey data to approximate the amount of earth to be moved. The definite integral can be used to derive formulas to make such calculations [3].

Usually, the integral formula, used for evaluating the area and volume, is simply memorized by students. If the conceptual base of integration has been well constructed, almost every formula of integral applications can be easily obtained.

\section{Methodology}

In this study, a semi-structured interview was carried out. In this interview, we tried to construct the disk method formula.

- Introducing the concept: evaluating the volume of an Egyptian pyramid.

- Evaluating the volume of a cone obtained by revolution (using Maple worksheet).

- Designing their own ring and evaluating its price (using Maplet).

The interview has been presented as a dialog between teacher and students. A CAS has been used to visualize the abstract three-dimensional concepts and to calculate some procedures. Our study involved a group of students at an average Turkish university. These students were taking an elective and elementary Maple course during the time of the implementation of the study. In this study, students were required to construct their own problem. We tried to get students to consider their own solutions by using a visualization created with Maple. This procedure was carried out by using a semi-structured interview. 


$$
\text { "actumen" — 2008/4/9 — 9:44 — page 367 — \#5 }
$$

Constructing the disk method formula for the volume obtained by revolving a curve ... $\quad 367$

\section{Pre-Concepts}

Following steps, which are used to construct the integral giving the volume of a three dimensional object, are assumed to be known by students:

- An interval $[a, b]$, on an axis, is being divided into $n$ equal parts. Let's call the width of every part $\Delta x_{n}$.

- $V_{n}$ represents $n$ times the volume that is obtained by multiplying $\Delta x_{n}$ and $A_{n}$, which is the cross-section area obtained by the same rule and is related to only one variable belonging to the interval $[a, b]$.

- Of course, for every three-dimensional shape, its volume is $\Delta x_{n} \cdot A_{n}$. This volume formula belongs to the object perpendicular to the cutting axis. Here, this axis has been assumed as $x$-axis.

- $\sum_{k=1}^{n} A_{k} \Delta x_{k}$ gives us the volume of an object like a kind of three-dimensional staircase.

- $\sum_{k=1}^{n} A_{k} \Delta x_{k}$ gives the volume of our shape approximately. We have to use the limit concept to find the exact volume like $\lim _{n \rightarrow \infty} \sum_{k=1}^{n} A_{k}(x) \Delta x_{k}$.

- Since $x$ variable varies in the interval $[a, b]$ above limit means $\int_{a}^{b} A(x) d x$.

\section{Presentation of Findings}

First, a problem was given to students:

Problem: Find a general formula for the volume of a square-based Egyptian pyramid by using the integral concept.

Key question: How can you divide the pyramid into regular cross-section areas, and how can you define these cross-section areas by in terms of the cutting variable?

Teacher: Consider a pyramid.

We obtain a cross-section area $A B C D$ by cutting the pyramid, perpendicular to its height.

Let one side of the base be $a,|O H|=h$ and let $|E H|=x$ and $|E F|=t$ be variables for the height and a side of the base, respectively. 

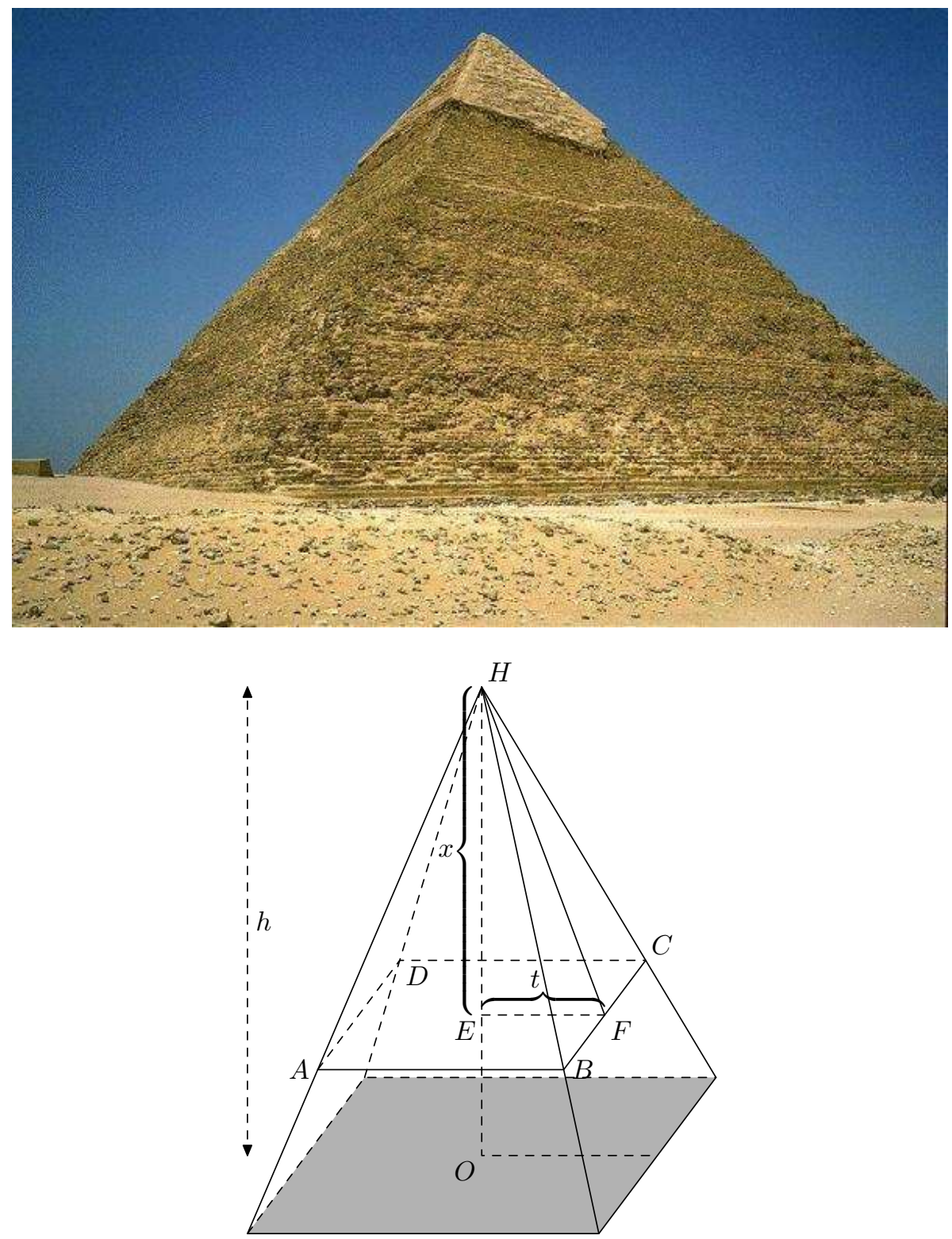

While the point $E$ is changing between the points $O$ and $H$, the area $A B C D$ changes regularly.

Teacher: Can you determine the area of $A B C D$ in terms of the variable $x$ ? 


$$
\text { "actumen" — 2008/4/9 — 9:44 — page 369 — \#7 }
$$

Constructing the disk method formula for the volume obtained by revolving a curve ... 369

Students: First, I think it is necessary to find a relationship between $x$ and $t$.

Let the angle $E H F$ be $\alpha$. Therefore $t=x \tan \alpha$.

Then, I can write the area $A B C D$ as $(2 t)^{2}=4 t^{2}$.

Last, I can define the area as $A(x)=4(x \tan \alpha)^{2}$.

Teacher: Suppose the area $A(x)$ has a little thickness $\Delta x$. Determine the volume of this box, whose base is the area $A(x)$ and height is $\Delta x$.

Students: Volume of Pyramid $=\lim _{\Delta x \rightarrow 0} \sum_{x \in[O, H]} A(x) \Delta x=\int_{0}^{h} A(x) d x$. $\int_{0}^{h} 4 x^{2} \tan ^{2} \alpha d x$.

Answer:

$$
V=\int_{0}^{h} 4 x^{2} \tan ^{2} \alpha d x=4 \tan ^{2} \alpha \int_{0}^{h} x^{2} d x=\left.4 \tan ^{2} \alpha \frac{x^{3}}{3}\right|_{0} ^{h}=4 \tan ^{2} \alpha \frac{h^{3}}{3} .
$$

Guide question for students: This result shows us how the volume is related the top angle of the pyramid. But we know the volume formula of a pyramid, and the formula is not related to this angle. How can you explain?

Answer:

Yes, it seems like that! But we can express the result as follows:

$$
\frac{a}{2}=h \tan \alpha \text { and } V=4 \tan ^{2} \alpha \frac{h^{3}}{3}=\frac{(2 h \tan \alpha)^{2} h}{3}=\frac{a^{2} h}{3}
$$

At this point, the students used Maple to better understand concept of approximations.

\section{Maple File Instructions}

In this worksheet, students determine the height of a cross section and, using Maple, draw cylinders that approximate the cross sections. Maple computes the sum of cylinders' volumes. Students can compare sum of the cylinders' volumes and volume of the cone (Table 1).

First, students determined the height of the cylinders. They entered the value as 5 . 
Then, they executed the command lines. Maple computed sum of the volumes of the cylinders. After computing the volume of the cone using Maple, students compared these results.

Second, students entered a new value for the height of the cylinders. They entered 2, 0.5, and 0.1 consecutively. In this way, students realized that if the height of the cylinders is decreased, the sum of the volumes of the cylinders will approximate the volume of the cone.

Table 1. Approximate to Volume of Cone

\begin{tabular}{|c|c|}
\hline \multicolumn{2}{|c|}{ Volume of Cone: $8000 \cdot \pi / 3=8377.580412$} \\
\hline 9676.105374 & $\begin{array}{c}\text { Approximate to Volume of Cone: } \\
\text { Approximate to Volume of Cone: }\end{array}$ \\
\hline 2094.357670 \\
\hline
\end{tabular}

Finally, students computed the volume of the cone like computing volume of the pyramid with paper and pencil method. This process is below.

Students: In general, we can call the term $A(x)$ in $\int_{0}^{h} A(x) d x$ as a crosssection area that depends on the variable $x$, which varies between 0 and $h$.

Let's define $A(x)$ for the cone:

Again the angle $\alpha$ is same angle as in pyramid, we can write $r=x \tan \alpha$ where $r$ is radius of a cross-section circle.

Therefore, $A(x)=\pi r^{2}=\pi(x \tan \alpha)^{2}$. 


$$
\text { "actumen" — 2008/4/9 — 9:44 — page 371 — \#9 }
$$

Constructing the disk method formula for the volume obtained by revolving a curve ... 371

Volume of The Cone $=\int_{0}^{h} A(x) d x=\int_{0}^{h} \pi x^{2} \tan ^{2} \alpha d x$, where $h$ is the height of the cone.

Volume of The Cone $=\int_{0}^{h} \pi x^{2} \tan ^{2} \alpha d x=\pi \tan ^{2} \alpha \int_{0}^{h} x^{2} d x=\left.\pi \tan ^{2} \alpha \frac{x^{3}}{3}\right|_{0} ^{h}=$ $\pi \tan ^{2} \alpha \frac{h^{3}}{3}$

Then, let's substitute $R=h \tan \alpha$, where $R$ is the radius of the base:

Volume of The Cone $=\pi \tan ^{2} \alpha \frac{h^{3}}{3}=\pi h \frac{(h \tan \alpha)^{2}}{3}=\frac{\pi h R^{2}}{3}$.

Teacher: Let's summarize our result! What is the main difference and similarity between two results?

Students: We have used the same integral, which is $\int_{0}^{h} A(x) d x$. In this integral, $A(x)$ is a function, defining the area of a cross-section area, which depends on $x$. Since, the cross-sections are different for the cone and pyramid, the function $A(x)$ was different in the two evaluations.

Teacher: OK! Let's define an integral formula for the volume, obtained by the revolution of any function around $x$-axis.

Students: When we revolve a part of the function $f(x)$ around the $x$-axis we will obtain a shape, whose cross-section area is a circle. This circle's radius is $f(x)$. So, we can define following formula:

$$
\pi \int_{a}^{b}[f(x)]^{2} d x
$$

If we revolve an area between the functions $f(x)$ and $g(x)$ around the $x$-axis, where $f(x) \geq g(x)$ for all $x \in[a, b]$, the volume will be $\pi \int_{a}^{b}\left[f(x)^{2}-g(x)^{2}\right] d x$.

At this point, the students used a Maplet to calculate and visualize the concept of volume.

You can find the Maple file for evaluating the volume of a cone and Maplet file for designing their own rings, prepared in Maple 9 at http://w3.gazi.edu . tr/web/aktumen/diskmethod/disk.htm. 


\section{Maplet Instructions}

With the help of this Maplet (Table 2), students benefited by:

- Visualizing the area, revolved around an axis and the volume obtained by revolution.

- Checking their evaluations.

- Researching how to find a specific shape by translating the functions along the $x$-axis or $y$-axis.

We have designed the Maplet having the capability to evaluate the area and volume between two curves. Students can evaluate the volume obtained by revolving only one curve around the $x$-axis by choosing second function as $y=0$.

We have given a project named "design your own ring and determine its price assuming that we know the price of the gold for unit volume."

Table 2. Maplet Screenshot

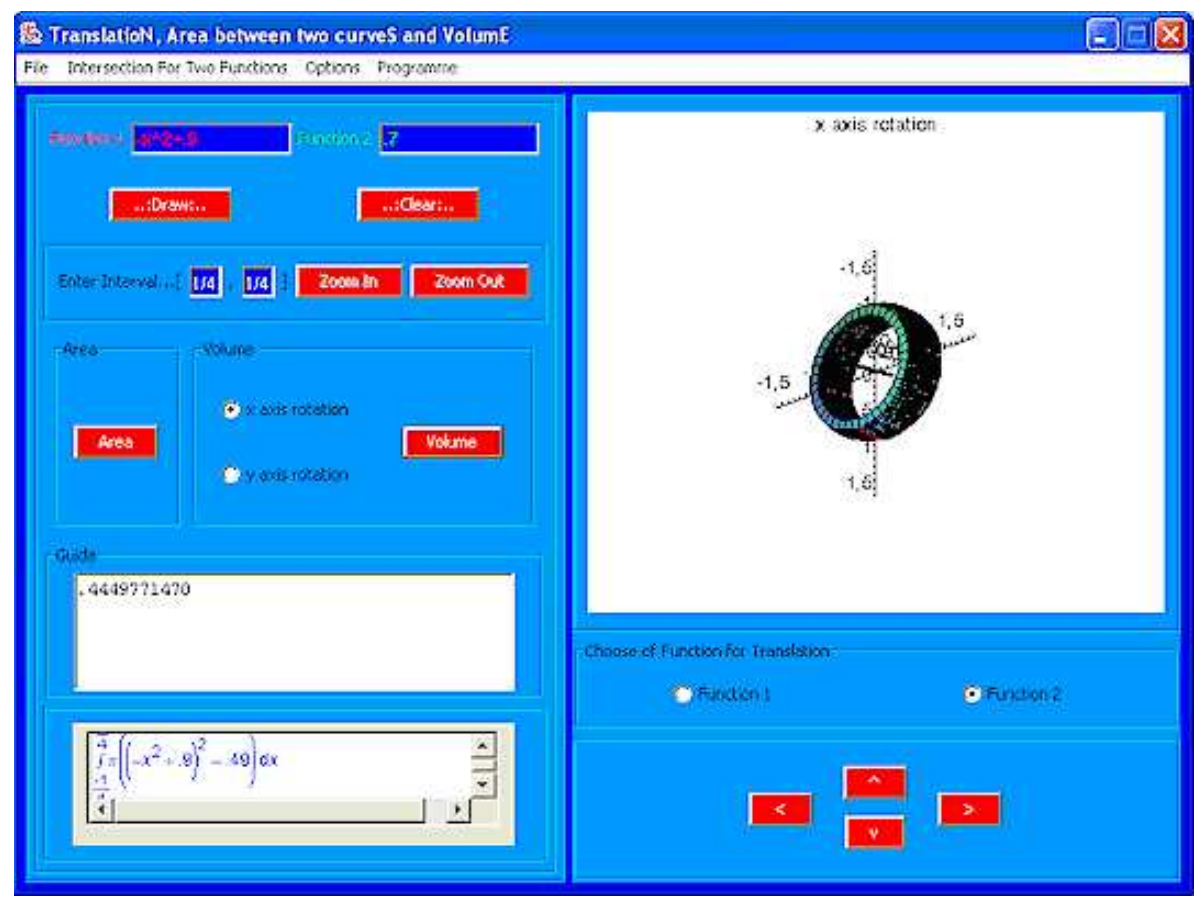




\section{Some of Rings Designed By Students}

The rings which were designed by students are in Table 3(a) and Table 3(b).

Table 3. Some of Ring Designed By Students $\left(0.1 \mathrm{br}^{3}=50\right.$ YTL $)$

(a)

- $f(x)=x^{2}+0.9$
- $g(x)=x^{2}+0.6$
- Interval: $[-0.3,0.3]$
- Volume: 0.8821
- Price: 441 YTL
- ${ }^{2}$ Price: $697.28 \mathrm{YTL}$
- $f(x)=x^{2}+0.8$
- Interval: $[-0.2,0.6]$
Volume: 1.39456


Table 3. Some of Ring Designed By Students

(b)

- $f(x)=0.4+\tan x$
- $g(x)=1.8-\tan x$
- Interval: $[3.6,3.9]$
- Volume: 0.46635
- Price: $233.17 \mathrm{YTL}$
- $f(x)=\cos (2 x)$
- $g(x)=\cos (2 x)+0.2$
- Interval: $[-0.3,0.3]$
- - Polume: 0.7849

\section{Conclusions}

In this study, the interview has been presented as a dialog between teacher and students. When we look at feedback from students, we see that such a teaching method effects students in a positive way and causes them to gain conceptual understanding directed towards the concepts of approximation and volume.

By using Maple, students have an active part in the learning environment. They have not only the chance to examine some situations with respect to the 


$$
\text { "actumen" — 2008/4/9 — 9:44 — page 375 — \#13 }
$$

Constructing the disk method formula for the volume obtained by revolving a curve ... 375

values the lecturer gives, but also the chance to examine graphics and results with respect to values they have determined themselves.

Starting the lesson with a real life problem attracts the students' attention and plays an important role in motivating them. Studying as a group enables students to share their opinions and to reach results through group discussion.

\section{References}

[1] R. Pierce, K. Stacey, Monitoring Effective Use of Computer Algebra Systems, in: Mathematics Education in the South Pacific, Proceedings of the $25^{\text {th }}$ Annual Conference of the Mathematics Education Research Group of Australasia, (B. Barton, K. C. Irwin, M. Pfannkuck \& M. O. J. Thomas, eds.), 2002.

[2] R. Clements, Essential Mathematical Concepts Needed by User of Computer Algebra, Teaching Mathematics and its Applications 18, no. 4 (1999).

[3] Midshipmen Online Calculus Labs, http://www .usna.edu/MathDept/website/courses/calc_labs/slicing/Application.html, access date: 05.02.2007.

[4] I. Cnop, New Insight in Mathematics by Live CAS Documents, Albuquerque Conference, 2001.

[5] N. Zehavi, Exploring the relationship between reflective thinking and execution in solving problems with a Computer Algebra System, International Workshop on research in Secondary and Tertiary Mathematics Education, 7-11 July, 2006.

[6] E. Dubinsky, K. Schwingendorf, Calculus, Concepts, Computers and Cooperative Learning (C4L), The Purdue Calculus Reform Project, 2004.

[7] C. Leinbach, D. C. Pountney and T. Etchells, Appropriate Use of a CAS in the Teaching and Learning of Mathematics, International Journal of Mathematical Education in Science and Technology 33, no. 1 (2002), 1-14.

[8] I. Malabar and D. C. Pountney, When is it appropriate to use of a Computer Algebra System (CAS)?, Proceedings of ICTMT4 Playmouth, 1999.

[9] U. Chundang, Using CAS for the visualization of some basic concepts in calculus of several variables, TCM Conference, Japan, 1998.

[10] E. Galindo, Visualization and Students' Performance in Technology based Calculus, $17^{\text {th }}$ PME-NA, Columbus, OH, October 21-24, 1995.

[11] S. Cunningham, Some strategies for using visualization in mathematics teaching, ZDM 3 (1994), 83-85.

[12] T. Kabaca and S. Mirasyedioglu, Constructing the Limit Concept by using a Computer Algebra System [CAS], International Conference of Teaching Mathematics (ICTM), 2006. 


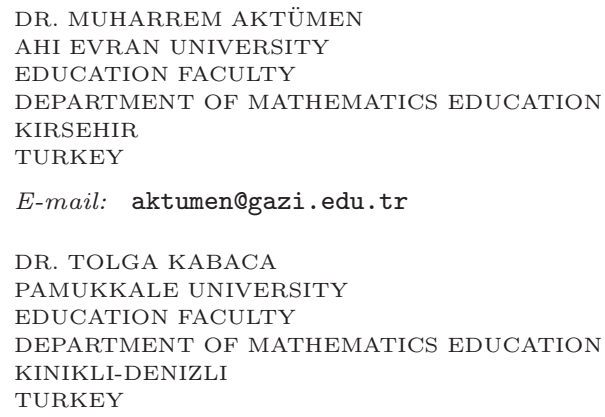

(Received July, 2007) 